\title{
Clinical and ethical perspectives of medical professionals towards female genital cosmetic procedures
}

\section{Tıp uzmanlarının genital kozmetik müdahalelere klinik ve etik açıdan bakış açıları}

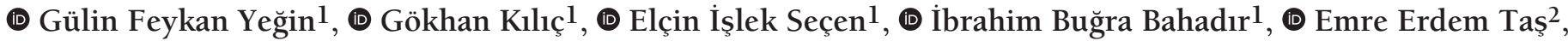 \\ ๑ Hüseyin Levent Keskin ${ }^{3}$, ๑ Ayşe Filiz Yavuz² \\ 1 Ankara City Hospital, Clinic of Gynecology and Obstetrics, Ankara, Turkey \\ ${ }^{2}$ Ylldırım Beyazıt University Faculty of Medicine, Department of Gynecology and Obstetrics, Ankara, Turkey \\ ${ }^{3}$ University of Health Sciences Turkey Faculty of Medicine, Department of Gynecology and Obstetrics, İstanbul, Turkey
}

\begin{abstract}
Objective: To evaluate the attitudes of medical students and professionals towards female genital cosmetic procedures (FGCPs) in terms of medical justification, applicability in practical life, ethical concerns, patient autonomy, and the clinical/social/psychological benefits-harms of these procedures.

Materials and Methods: A semi-structured questionnaire providing information about the attitudes of medical students and specialists (n=623) towards FGCPs including G-spot amplification, clitoral hood reduction, vaginoplasty, labia majora augmentation/reduction, labia minora augmentation/reduction, hymenoplasty, laser procedures, vulvar/perianal bleaching, and liposculpture, was completed by a target population and evaluated statistically.

Results: Participants stated that FGCPs could be performed only upon patient request and there could rarely be a medical indication for their performance $(\mathrm{p}<0.05)$. Nearly half $(44.5 \%)$ of the participants regarded hymenoplasty as controversial in terms of ethical issues, and $44.6 \%$ of participants do so for G-spot amplification. Over half (54.5\%) of the participants agreed on the positive effect of FGCPs on improving the quality of life, 55.4\% on improving self-esteem, and $54.1 \%$ on improving sexual functions of women. About half (49.3\%) of respondents thought that the advertising and encouragement of FGCPs should be forbidden and $47 \%$ were indecisive about whether FGCPs constituted genital mutilation.

Conclusion: The majority of the participants declared that FGCPs could be performed only upon patient request and improve self-esteem, quality of life, and sexual functions. The most controversial procedures in terms of ethics were hymenoplasty and G-spot amplification. Detailed guidelines for the protection of both patients and physicians are needed because the recommendations on FGCPs are insufficient to define the boundaries of medical justification, genital mutilation, advertising, and ethical concerns.

Keywords: Cosmetic surgery, ethics, G-spot, hymenoplasty, vaginoplasty

$\ddot{\mathrm{O} z}$

Amaç: Tıp öğrencilerinin ve profesyonellerin kadın genital kozmetik prosedürlerine (KGKP) tıbbi gerekçelendirme, pratik hayatta uygulanabilirliği, etik kaygılar, hasta otonomisi ve prosedürlerin klinik/sosyal/psikolojik yararları-zararları açısından bakış açılarını değerlendirmek.

Gereç ve Yöntemler: Tıp ögrencileri ve uzmanların (n=623) G-noktası augmentasyonu, klitoral hudoplasti, vajinoplasti, labia majora büyütme/küçültme, labia minora büyütme/küçültme, himenoplasti, lazer prosedürleri, vulvar/perianal beyazlatma ve liposculpturing dahil olmak üzere KGKP'lere yönelik tutumları hakkında bilgi veren bir anket hedef popülasyona uygulanmış ve istatistiksel olarak değerlendirilmiştir.

Bulgular: Katılımcılar, KGKP’lerin yalnızca hasta talebi üzerine gerçekleştirilebileceğini ve nadiren prosedürün tıbbi bir endikasyonu olabileceğini belirtti (p<0,05). Katılımcıların \%44,5'i himenoplastiyi etik açıdan tartışmalı bulurken, katılımcıların \%44,6'sı aynı yorumu G-noktası amplifikasyonu için yapmaktadır. Katılımcıların \%54,5’i, KGKP'lerin yaşam kalitesini iyileştirme, \%55,4'ü benlik saygısı geliştirme ve \%54,l’i kadınların cinsel işlevlerini iyileştirme üzerindeki pozitif etkisi konusunda hemfikirdir. Ankete katılanların \%49,3’ü, KGKP'ler ile ilgili reklam stratejilerinin ve teşviklerinin yasaklanması gerektiğini düşünürken, \%47’si KGKP'lerin genital mutilasyon olarak görülmesi konusunda kararsızdı.
\end{abstract}

PRECIS: By using a self-administered questionnaire, we evaluated the clinical, ethical, and sociocultural perspectives of medical professionals and students towards female genital cosmetic procedures.

Address for Correspondence/Yazıșma Adresi: Gülin Feykan Yeğin MD,

Ankara City Hospital, Clinic of Gynecology and Obstetrics, Ankara, Turkey

Phone: +90 5072479592 E-mail: gulin_yegin@hotmail.com ORCID ID: orcid.org/0000-0001-8006-5055

Received/Geliş Tarihi: 09.01.2021 Accepted/Kabul Tarihi: 02.04.2021

${ }^{\oplus}$ Copyright 2021 by Turkish Society of Obstetrics and Gynecology

Turkish Journal of Obstetrics and Gynecology published by Galenos Publishing House. 
Sonuç: Katılımcıların büyük çoğunluğu, KGKP’lerin sadece hastanın isteği üzerine yapılabileceğini ve benlik saygısını, yaşam kalitesini ve cinsel fonksiyonları iyileştirdiğini belirtmiştir. Etik açıdan en tartışmalı prosedürlerin, kızlık zarı dikimi ve G-noktası amplifikasyonu olduğu bildirildi. KGKP'ye yönelik kılavızlar tıbbi gerekçelendirme, genital mutilasyon, reklam ve etik kaygıların sınırlarını tanımlamak için yetersiz olduğundan; hem hastaların hem de doktorların korunması için ayrıntılı kılavuzlara ihtiyaç vardır.

Anahtar Kelimeler: Kozmetik cerrahi, etik, G-noktası, himenoplasti, labioplasti, vajinoplasti

\section{Introduction}

The term "female genital cosmetic procedures" (FGCPs) encompasses numerous interventions, including surgeries (G-spot amplification, labia majora augmentation, labia majora reduction, labia minora augmentation, labia majora reduction, clitoral hood reduction, vaginoplasty, and hymenoplasty) and non-surgical procedures (vulvar/perianal bleaching/ whitening, liposculpture, laser for vaginal tightening, and laser for genitourinary syndrome of menopause). Although there is increasing popularity, patient demand and performance rate, ethical and safety concerns have been raised about the performance of FGCPs. The perception of "genital beautification" augmented by the Internet and media forces, caused women to fail in the decision of whether her vulvar image was normal ${ }^{(1,2)}$. Bioethical analysis of cosmetic surgery revives several controversial issues regarding the principles of ethical medical care $^{(3)}$. The ethical concept of beneficence and non-maleficence has been forcing authorities to question the ethics of undergoing surgical risk to improve the physical appearance. In addition to medical objections, many critics are concerned about the social and cultural aspects of cosmetic surgery ${ }^{(3,4)}$. The results of a survey could be useful in determining clinical strategies regarding FGCPs in terms of health policies.

The goals of this survey were to analyze the attitudes of medical professionals and students towards FGCPs in terms of medical justification, applicability in practical life, ethical concerns, patient autonomy, and the clinical/social/psychological benefitsharms of these procedures.

\section{Materials and Methods}

This cross-sectional study was performed via a web-based, semi-structured questionnaire. Forms were collected between December $15^{\text {th }}, 2019$, and March $30^{\text {th }}, 2020$. The study was approved by the Institutional Review Board (E1/180/2019). The respondents were informed and consent for participation was obtained before administering the questionnaire.

The survey form was planned after a comprehensive review of the literature including medical indications, ethical issues, and controversial issues regarding esthetic gynecologic procedures ${ }^{(5,6)}$

The survey consisted of questions for 12 FGCPs (G-spot amplification, clitoral hood reduction, labia majora augmentation, labia majora reduction, labia minora augmentation, labia majora reduction, laser vaginal tightening, laser for genitourinary syndrome of menopause, vaginoplasty, vulvar/perianal bleaching, liposculpture and hymenoplasty) including:
1. First section: The demographics of the participants [age, sex, differentiation (students, specialists), speciality].

2. Second section: Participants' opinion about the existence of "G-spot" and ethical issues regarding hymenoplasty - 3 questions, 3-point Likert scale including answers: "agree", "indecisive", and "disagree".

3. Third section: The participants were questioned about whether the procedure was medically justifiable, and could be performed with only patient demand - 2 questions for 12 FGCPs separately, 3-point Likert for asking medical justification including answers "never/rarely/often" or 2-point Likert scale for others including answers "yes/no".

4. Fourth section: Participants were asked whether the procedures were "ethical", "unethical" or "debatable" in terms of medical ethics. Participants answered question separately for each of 12 FGCPs.

5. Fifth section: Participants opinions were asked about given speculative comments regarding patient selection criteria, age limit and potential benefits/harms - 13 questions were evaluated using 3-point Likert with answers: "agree", "indecisive", and "disagree".

The link of the questionnaire was sent to the target population via email (collected from the databases of medical societies), and also posted in specific social network groups for physicians/ medical students. The data of the respondents were collected automatically through a web-based system (https://docs.google. com/forms, CA, USA). Statistical power analysis was performed before applying to the ethics committee, which showed that a sample of 387 would be enough to achieve a confidence level of $95 \%$ and a confidence interval of $5 \%$ according to the estimated size of the population of physicians and medical students in our country.

\section{Statistical Analysis}

The statistical analysis was performed using the Statistical Package for the Social Sciences (SPSS) software, version 20.0 (IBM Corp., Armonk, NY, USA). The chi-square test was used for the analysis of variables, and $\mathrm{p}<0.05$ was considered statistically significant.

\section{Results}

\section{Characteristics of Participants}

The number of participants who received the survey was 623 . One hundred twenty of the respondents were medical students/ residents ( $81 \%$ were residents). Specialists were classified into four groups as follows: obstetrics and gynecology $(n=183$, $37 \%)$, general practitioners $(n=101,20 \%)$, other surgical 
( $\mathrm{n}=117,23 \%)$, and other non-surgical $(\mathrm{n}=102,20 \%)$. Two hundred sixty-five (42.5\%) of the participants were male and 358 (57.5\%) were female. Assistants (speciality trainees) were also included in the specialists' group. All participants were working in public hospitals, and 243 (39\%) were lecturers in universities. Two hundred twenty-nine (36.7\%) respondents were aged $\leq 30$ years, $186(29.8 \%)$ were aged between 31 and 40 years, 160 (25.6\%) were aged 41-50 years, and 49 participants were aged $\geq 51$ years. Eighty-eight $(14.1 \%)$ respondents planned to undergo plastic surgery and $36(5.8 \%)$ had undergone at least one plastic surgery.

\section{Results of Survey}

Almost half (49.3\%) of the participants found reasonable that a woman's need for hymenoplasty originated from social oppression (Figure 1). Differentiation (student/specialist) and sex was not an identifier on this statement. Most (63.2\%) of participants agreed that women who are in demand should have hymenoplasty (Figure 1). One-third (33.9\%) of the participants stated that performing hymenoplasty had no indications ever and this opinion was more common among specialists (Table 1). The opinion of the participants about hymenoplasty was evaluated as ethical, unethical or debatable in terms of ethics and the ratios were $40.3 \%, 15.2 \%$, and $44.5 \%$, respectively. The statement that hymenoplasty was controversial in terms of medical ethics was higher among females compared with males $(p<0.05)$. Details of data regarding ethical perceptions are given in Figure 2 and Table 2. One-quarter (24.1\%) of the participants considered that the G-spot existed (Figure 1); $26.4 \%$ of the females and $22.3 \%$ of the males agreed on

Table 1. Opinions of specialists on the medical justification of female genital cosmetic procedures

\begin{tabular}{|c|c|c|c|c|c|c|c|c|c|c|c|c|c|}
\hline & \multicolumn{4}{|c|}{ Never $\%(\mathrm{n} / \mathrm{N})$} & \multicolumn{4}{|c|}{ Rarely\% (n/N) } & \multicolumn{4}{|c|}{ Often $\%(\mathrm{n} / \mathrm{N})$} & \multirow[b]{2}{*}{ a } \\
\hline & 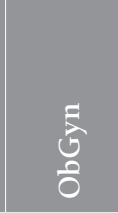 & ثิ & 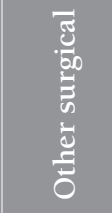 & 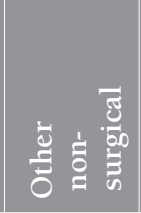 & \begin{tabular}{l}
\multirow{2}{0}{} \\
0 \\
0
\end{tabular} & 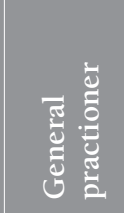 & 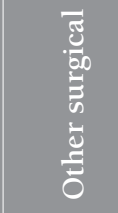 & 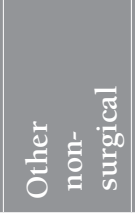 & $\begin{array}{l}\text { స్ } \\
\text { ह } \\
0\end{array}$ & 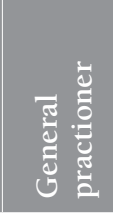 & 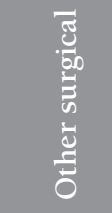 & 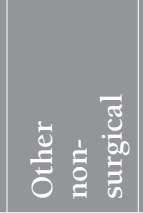 & \\
\hline $\begin{array}{l}\text { G-spot } \\
\text { amplification }\end{array}$ & $\begin{array}{l}39.3 \% \\
72 / 183\end{array}$ & $\begin{array}{l}34.7 \% \\
35 / 101\end{array}$ & $\begin{array}{l}25.6 \% \\
30 / 117\end{array}$ & $\begin{array}{l}25.5 \% \\
26 / 102\end{array}$ & $\begin{array}{l}43.2 \% \\
79 / 183\end{array}$ & $\begin{array}{l}57.4 \% \\
58 / 101\end{array}$ & $\begin{array}{l}64.1 \% \\
75 / 117\end{array}$ & $\begin{array}{l}66.7 \% \\
68 / 102\end{array}$ & $\begin{array}{l}17.5 \% \\
32 / 183\end{array}$ & $\begin{array}{l}7.9 \% \\
8 / 101\end{array}$ & $\begin{array}{l}10.3 \% \\
12 / 117\end{array}$ & $\begin{array}{l}7.8 \% \\
8 / 102\end{array}$ & $<0.01^{*}$ \\
\hline $\begin{array}{l}\text { Clitoral hood } \\
\text { reduction }\end{array}$ & $\begin{array}{l}22.4 \% \\
41 / 183\end{array}$ & $\begin{array}{l}30.7 \% \\
31 / 101\end{array}$ & $\begin{array}{l}17.9 \% \\
21 / 117\end{array}$ & $\begin{array}{l}29.4 \% \\
30 / 102\end{array}$ & $\begin{array}{l}57.9 \% \\
106 / 183\end{array}$ & $\begin{array}{l}60.4 \% \\
61 / 101\end{array}$ & $\begin{array}{l}70.1 \% \\
82 / 117\end{array}$ & $\begin{array}{l}60.8 \% \\
62 / 102\end{array}$ & $\begin{array}{l}19.7 \% \\
36 / 183\end{array}$ & $\begin{array}{l}8.9 \% \\
9 / 101\end{array}$ & $\begin{array}{l}12 \% \\
14 / 117\end{array}$ & $\begin{array}{l}9.8 \% \\
10 / 102\end{array}$ & $0.02 *$ \\
\hline Hymenoplasty & $\begin{array}{l}38.8 \% \\
71 / 183\end{array}$ & $\begin{array}{l}34.7 \% \\
35 / 101\end{array}$ & $\begin{array}{l}36.8 \% \\
43 / 117\end{array}$ & $\begin{array}{l}36.3 \% \\
37 / 102\end{array}$ & $\begin{array}{l}41 \% \\
75 / 183\end{array}$ & $\begin{array}{l}57.4 \% \\
58 / 101\end{array}$ & $\begin{array}{l}50.4 \% \\
59 / 117\end{array}$ & $\begin{array}{l}53.9 \\
55 / 102\end{array}$ & $\begin{array}{l}20.2 \% \\
37 / 183\end{array}$ & $\begin{array}{l}7.9 \% \\
8 / 101\end{array}$ & $\begin{array}{l}12.8 \% \\
15 / 117\end{array}$ & $\begin{array}{l}9.8 \% \\
10 / 102\end{array}$ & $0.03 *$ \\
\hline $\begin{array}{l}\text { Labia majora } \\
\text { augmentation }\end{array}$ & $\begin{array}{l}25.1 \% \\
46 / 183\end{array}$ & $\begin{array}{l}28.7 \% \\
29 / 101\end{array}$ & $\begin{array}{l}26.5 \% \\
31 / 117\end{array}$ & $\begin{array}{l}24.5 \% \\
25 / 102\end{array}$ & $\begin{array}{l}55.2 \% \\
101 / 183\end{array}$ & $\begin{array}{l}62.4 \% \\
63 / 101\end{array}$ & $\begin{array}{l}59.8 \% \\
70 / 117\end{array}$ & $\begin{array}{l}67.6 \% \\
69 / 102\end{array}$ & $\begin{array}{l}19.7 \% \\
36 / 183\end{array}$ & $\begin{array}{l}8.9 \% \\
9 / 101\end{array}$ & $\begin{array}{l}13.7 \% \\
16 / 117\end{array}$ & $\begin{array}{l}7.8 \% \\
8 / 102\end{array}$ & 0.08 \\
\hline $\begin{array}{l}\text { Labia majora } \\
\text { reduction }\end{array}$ & $\begin{array}{l}18 \% \\
33 / 183\end{array}$ & $\begin{array}{l}26.7 \% \\
27 / 101\end{array}$ & $\begin{array}{l}18.8 \% \\
22 / 117\end{array}$ & $\begin{array}{l}26.5 \% \\
27 / 102\end{array}$ & $\begin{array}{l}59.6 \% \\
109 / 183\end{array}$ & $\begin{array}{l}65.3 \% \\
66 / 101\end{array}$ & $\begin{array}{l}66.7 \% \\
78 / 117\end{array}$ & $\begin{array}{l}65.7 \% \\
67 / 102\end{array}$ & $\begin{array}{l}22.4 \% \\
41 / 183\end{array}$ & $\begin{array}{l}7.9 \% \\
8 / 101\end{array}$ & $\begin{array}{l}14.5 \% \\
17 / 117\end{array}$ & $\begin{array}{l}7.8 \% \\
8 / 102\end{array}$ & 0.01 \\
\hline $\begin{array}{l}\text { Labia minora } \\
\text { augmentation }\end{array}$ & $\begin{array}{l}33.3 \% \\
61 / 183\end{array}$ & $\begin{array}{l}28.7 \% \\
29 / 101\end{array}$ & $\begin{array}{l}23.9 \% \\
28 / 117\end{array}$ & $\begin{array}{l}26.5 \% \\
27 / 102\end{array}$ & $\begin{array}{l}46.4 \% \\
85 / 183\end{array}$ & $\begin{array}{l}63.4 \% \\
64 / 101\end{array}$ & $\begin{array}{l}58.1 \% \\
68 / 117\end{array}$ & $\begin{array}{l}65.7 \% \\
67 / 102\end{array}$ & $\begin{array}{l}20.2 \% \\
37 / 183\end{array}$ & $\begin{array}{l}7.9 \% \\
8 / 101\end{array}$ & $\begin{array}{l}17.9 \% \\
21 / 117\end{array}$ & $\begin{array}{l}7.8 \% \\
8 / 102\end{array}$ & $<0.01 *$ \\
\hline $\begin{array}{l}\text { Labia minora } \\
\text { reduction }\end{array}$ & $\begin{array}{l}14.2 \% \\
26 / 183\end{array}$ & $\begin{array}{l}24.8 \% \\
25 / 101\end{array}$ & $\begin{array}{l}24.8 \% \\
29 / 117\end{array}$ & $\begin{array}{l}28.4 \\
29 / 102\end{array}$ & $\begin{array}{l}57.9 \% \\
106 / 183\end{array}$ & $\begin{array}{l}68.3 \% \\
69 / 101\end{array}$ & $\begin{array}{l}61.5 \% \\
72 / 117\end{array}$ & $\begin{array}{l}63.7 \% \\
65 / 102\end{array}$ & $\begin{array}{l}27.9 \% \\
51 / 183\end{array}$ & $\begin{array}{l}6.9 \% \\
7 / 101\end{array}$ & $\begin{array}{l}13.7 \% \\
16 / 117\end{array}$ & $\begin{array}{l}7.8 \% \\
8 / 102\end{array}$ & $<0.01^{*}$ \\
\hline Vaginoplasty & $\begin{array}{l}3.8 \% \\
7 / 18\end{array}$ & $\begin{array}{l}19.8 \% \\
20 / 101\end{array}$ & $\begin{array}{l}8.5 \% \\
10 / 117\end{array}$ & $\begin{array}{l}23.5 \% \\
24 / 102\end{array}$ & $\begin{array}{l}55.2 \% \\
101 / 183\end{array}$ & $\begin{array}{l}69.3 \% \\
70 / 101\end{array}$ & $\begin{array}{l}66.7 \% \\
78 / 117\end{array}$ & $\begin{array}{l}66.7 \% \\
68 / 102\end{array}$ & $\begin{array}{l}41 \% \\
75 / 183\end{array}$ & $\begin{array}{l}10.9 \% \\
11 / 101\end{array}$ & $\begin{array}{l}24.8 \% \\
29 / 117\end{array}$ & $\begin{array}{l}9.8 \% \\
10 / 102\end{array}$ & $<0.01^{*}$ \\
\hline $\begin{array}{l}\text { Laser vaginal } \\
\text { tightening }\end{array}$ & $\begin{array}{l}18.6 \% \\
34 / 183\end{array}$ & $\begin{array}{l}25.7 \% \\
26 / 101\end{array}$ & $\begin{array}{l}21.4 \% \\
25 / 117\end{array}$ & $\begin{array}{l}24.5 \% \\
25 / 102\end{array}$ & $\begin{array}{l}56.8 \% \\
104 / 183\end{array}$ & $\begin{array}{l}63.4 \% \\
64 / 101\end{array}$ & $\begin{array}{l}60.7 \% \\
71 / 117\end{array}$ & $\begin{array}{l}67.6 \% \\
69 / 102\end{array}$ & $\begin{array}{l}24.6 \% \\
45 / 183\end{array}$ & $\begin{array}{l}10.9 \% \\
11 / 101\end{array}$ & $\begin{array}{l}17.9 \% \\
21 / 117\end{array}$ & $\begin{array}{l}7.8 \% \\
8 / 102\end{array}$ & $0.01^{*}$ \\
\hline Laser for GSM & $\begin{array}{l}15.3 \\
28 / 183\end{array}$ & $\begin{array}{l}22.8 \\
23 / 101\end{array}$ & $\begin{array}{l}17.9 \% \\
21 / 117\end{array}$ & $\begin{array}{l}23.5 \% \\
24 / 102\end{array}$ & $\begin{array}{l}57.9 \% \\
106 / 183\end{array}$ & $\begin{array}{l}66.3 \% \\
67 / 101\end{array}$ & $\begin{array}{l}62.4 \% \\
73 / 117\end{array}$ & $\begin{array}{l}66.7 \% \\
68 / 102\end{array}$ & $\begin{array}{l}26.8 \% \\
49 / 183\end{array}$ & $\begin{array}{l}10.9 \% \\
11 / 101\end{array}$ & $\begin{array}{l}19.7 \% \\
23 / 117\end{array}$ & $\begin{array}{l}9.8 \% \\
10 / 102\end{array}$ & $0.01^{*}$ \\
\hline $\begin{array}{l}\text { Vulvar/ } \\
\text { perianal } \\
\text { bleaching }\end{array}$ & $\begin{array}{l}32.2 \% \\
59 / 183\end{array}$ & $\begin{array}{l}31.7 \% \\
32 / 101\end{array}$ & $\begin{array}{l}25.6 \% \\
30 / 117\end{array}$ & $\begin{array}{l}29.4 \% \\
30 / 102\end{array}$ & $\begin{array}{l}46.4 \% \\
85 / 183\end{array}$ & $\begin{array}{l}60.4 \% \\
61 / 101\end{array}$ & $\begin{array}{l}59 \% \\
69 / 117\end{array}$ & $\begin{array}{l}62.7 \% \\
64 / 102\end{array}$ & $\begin{array}{l}21.3 \% \\
39 / 183\end{array}$ & $\begin{array}{l}7.9 \% \\
8 / 101\end{array}$ & $\begin{array}{l}15.4 \% \\
18 / 117\end{array}$ & $\begin{array}{l}7.8 \% \\
8 / 102\end{array}$ & $0.01^{*}$ \\
\hline Liposculpture & $\begin{array}{l}32.8 \% \\
60 / 183\end{array}$ & $\begin{array}{l}28.7 \% \\
29 / 101\end{array}$ & $\begin{array}{l}34.2 \\
40 / 117\end{array}$ & $\begin{array}{l}25.5 \% \\
26 / 102\end{array}$ & $\begin{array}{l}49.2 \% \\
90 / 183\end{array}$ & $\begin{array}{l}64.4 \% \\
65 / 101\end{array}$ & $\begin{array}{l}53.8 \% \\
63 / 117\end{array}$ & $\begin{array}{l}66.7 \% \\
68 / 102\end{array}$ & $\begin{array}{l}18 \% \\
33 / 183\end{array}$ & $\begin{array}{l}6.9 \% \\
7 / 101\end{array}$ & $\begin{array}{l}12 \% \\
14 / 117\end{array}$ & $\begin{array}{l}7.8 \% \\
8 / 102\end{array}$ & $0.02 *$ \\
\hline
\end{tabular}

${ }^{a} G S M$ : Genitourinary syndrome of menopause, *statistically significant 


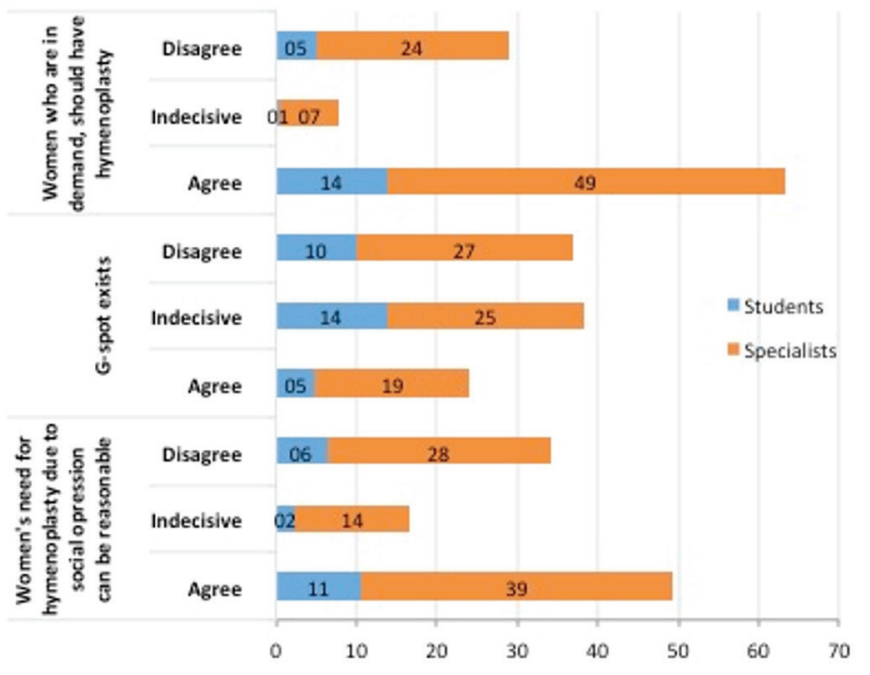

Figure 1. Expression of participants' thoughts about the existence of "G-spot" and ethical issues regarding hymenoplasty as percentages

the existence of the G-spot. In the entire cohort, 184 (29.5\%) participants stated that there was no medical indication to perform G-spot amplification. Details about specialities are given in Table 1. Just over half (53\%) of the entire cohort considered that G-spot amplification could be performed only upon patient request. The sex of the participants did not affect the attitude regarding G-spot procedures. Indecision about the ethical issues in G-spot amplification was higher among females $(\mathrm{p}<0.05)$. Details of data regarding ethical perceptions are given in Table 2 and Figure 2.

In the entire cohort, participants stated that all FGCPs could "rarely" be performed with a medical indication. The rate of answers that there was never a medical reason to perform the procedures was significantly higher for specialists compared with students, except for vaginoplasty $(\mathrm{p}<0.05)$. Surgeons (gynecologists and other surgical specialities) were more likely to think that labia majora reduction, labia minora augmentation, and vaginal laser procedures could be performed often with a medical indication (Table 1). The sex of the participants did not affect the opinion on medical indications of other cosmetic gynecologic procedures, except being a male was associated with the consideration of labia minora augmentation could often be performed with a medical indication (13.1\% vs 19.6; $\mathrm{p}<0.05$ ). For all cosmetic gynecologic procedures, most of the participants considered that it could be appropriate to perform surgery only upon patient request (53\% for G-spot amplification, 74\% for clitoral hood reduction, $67 \%$ for hymenoplasty, $77 \%$ for labia major augmentation, $79 \%$ for labia majora reduction, $76 \%$ for labia minora augmentation, $78 \%$ for labia majora reduction, $81 \%$ for vaginoplasty, $78 \%$ for laser procedures, $78 \%$ for bleaching and $76 \%$ for liposculpture). Agreement on this statement was significantly low in specialist groups compared with the students. Typically, the ratio of the agreement on patient autonomy was significantly lower in the non-surgical specialist and general practitioner groups $(\mathrm{p}<0.05)$. In questioning procedures in terms of ethical principles, the most frequent answer was "ethical" (Table 2). Sex did not affect the ethical view of the participants, except for vulvar bleaching; being male was related to thinking that vulvar bleaching was unethical (6.8\% vs $1.7 \%$; $\mathrm{p}<0.05$ ). Details of data on ethical perceptions are given in Table 2 and Figure 2. The majority (80.1\%) of the participants stated that FGCPs should not be performed on girls aged under 18 years. More than half $(56.3 \%)$ of the respondents agreed that FGCPs should be treated similarly to the surgeries at any anatomic site. Disagreement on this topic was most common among gynecologists and statistically significant, followed by the non-surgical specialities, general practitioners, and other surgical specialities, and the ratios were $50.5 \%, 27.1 \%, 13.1 \%$, and $9.3 \%$, respectively. The ratio of the participants who thought that the patient should be evaluated by a psychiatrist before undergoing surgery was $44.8 \%$. The disagreement rate on psychiatric evaluation was significantly higher among gynecologists (58.3\% of gynecologists, $8.6 \%$ of general practitioners, $13.7 \%$ of other surgeons, $19.4 \%$ of physicians in the non-surgical group; $\mathrm{p}<0.05)$.

One fifth $(20.7 \%)$ of the participants stated that the procedures should not be performed in public hospitals and 49.3\% thought that advertising and encouragement of FGCPs should be forbidden. Just under half (47\%) were indecisive about the evaluation of FGCPs as genital mutilation. Indecision was more common among specialists. About one-fifth (19.1\%) of physicians stated that FGCPs should be considered as genital mutilation and the rate of this statement was highest among gynecologists compared with other specialities $(49.5 \%$ of gynecologists, $8.1 \%$ of general practitioners, $26.3 \%$ of other surgeons, $16.2 \%$ of physicianss in the non-surgical group; $\mathrm{p}<0.05)$.

Just over half (54.5\%) of the participants agreed on the FGCPs effect on improving the quality of life, $55.4 \%$ on improving selfesteem, and $54.1 \%$ on improving sexual functions of women. While $25.4 \%$ of the study group considered that FGCPs were a temporary trend, $32.6 \%$ thought the opposite (Table 3).

The participants were indecisive about whether these procedures would improve dyspareunia and urinary incontinence, yet the disagreement rate was highest among gynecologists (dyspareunia; $65.5 \%$ of gynecologists, $17.1 \%$ of general practitioners, $5.3 \%$ of other surgeons, $11.8 \%$ of physicians in the non-surgical group; $\mathrm{p}<0.05$ ) (urinary incontinence; $66 \%$ of gynecologists, $14.4 \%$ of general practitioners, $10.3 \%$ of other surgeons, 9.3 of physicians in the non-surgical group; $\mathrm{p}<0.05)$.

\section{Discussion}

The current survey showed that the majority of the participants considered that FGCPs were appropriate to perform only upon patient request $(\mathrm{p}<0.05)$. Procedures considered to be the most controversial in terms of ethics were hymenoplasty and G- spot 
Table 2. Perceptions of specialists about ethical perspectives of female genital cosmetic procedures

\begin{tabular}{|c|c|c|c|c|c|c|c|c|c|c|c|c|c|}
\hline & \multicolumn{4}{|c|}{ Ethical \% (n/N) } & \multicolumn{4}{|c|}{$\begin{array}{l}\text { Debatable in terms of medical } \\
\text { ethics } \%(n / N)\end{array}$} & \multicolumn{4}{|c|}{ Unethical \% (n/N) } & \multirow[b]{2}{*}{ 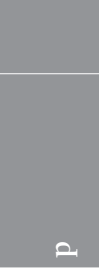 } \\
\hline & \begin{tabular}{l}
\multirow{5}{0}{} \\
0 \\
0
\end{tabular} & 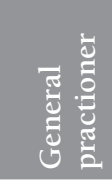 & 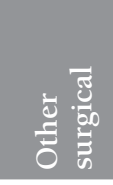 & 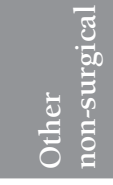 & $\begin{array}{l}\frac{1}{6} \\
0\end{array}$ & 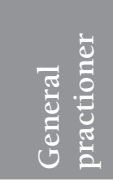 & 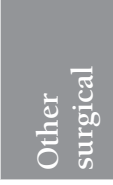 & 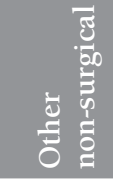 & \begin{tabular}{l}
\multirow{2}{0}{} \\
0 \\
0
\end{tabular} & 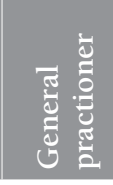 & 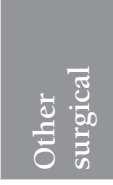 & 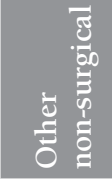 & \\
\hline $\begin{array}{l}\text { G-spot } \\
\text { amplification }\end{array}$ & $\begin{array}{l}42.1 \% \\
77 / 183\end{array}$ & $\begin{array}{l}30.7 \% \\
31 / 101\end{array}$ & $\begin{array}{l}65.8 \% \\
77 / 117\end{array}$ & $\begin{array}{l}34.3 \% \\
35 / 102\end{array}$ & $\begin{array}{l}45.4 \% \\
83 / 183\end{array}$ & $\begin{array}{l}60.4 \% \\
61 / 101\end{array}$ & $\begin{array}{l}26.5 \% \\
31 / 117\end{array}$ & $\begin{array}{l}61.8 \% \\
63 / 102\end{array}$ & $\begin{array}{l}12.6 \% \\
23 / 183\end{array}$ & $\begin{array}{l}8.9 \% \\
9 / 101\end{array}$ & $\begin{array}{l}7.7 \% \\
9 / 117\end{array}$ & $\begin{array}{l}3.9 \% \\
4 / 102\end{array}$ & $<0.01$ * \\
\hline $\begin{array}{l}\text { Clitoral hood } \\
\text { reduction }\end{array}$ & $\begin{array}{l}69.4 \% \\
127 / 183\end{array}$ & $\begin{array}{l}33.7 \% \\
34 / 101\end{array}$ & $\begin{array}{l}74.4 \% \\
87 / 117\end{array}$ & $\begin{array}{l}38.2 \% \\
39 / 102\end{array}$ & $\begin{array}{l}28.4 \% \\
52 / 183\end{array}$ & $\begin{array}{l}56.4 \% \\
57 / 101\end{array}$ & $\begin{array}{l}15.4 \% \\
18 / 117\end{array}$ & $\begin{array}{l}59.8 \% \\
61 / 102\end{array}$ & $\begin{array}{l}2.2 \% \\
4 / 183\end{array}$ & $\begin{array}{l}9.9 \% \\
10 / 101\end{array}$ & $\begin{array}{l}10.3 \% \\
12 / 117\end{array}$ & $\begin{array}{l}2 \% \\
2 / 102\end{array}$ & $<0.01 *$ \\
\hline Hymenoplasty & $\begin{array}{l}47 \% \\
86 / 183\end{array}$ & $\begin{array}{l}24.8 \% \\
25 / 101\end{array}$ & $\begin{array}{l}44.4 \% \\
52 / 117\end{array}$ & $\begin{array}{l}32.4 \% \\
33 / 102\end{array}$ & $\begin{array}{l}36.1 \% \\
66 / 183\end{array}$ & $\begin{array}{l}62.4 \% \\
63 / 101\end{array}$ & $\begin{array}{l}38.5 \% \\
45 / 117\end{array}$ & $\begin{array}{l}48 \% \\
49 / 102\end{array}$ & $\begin{array}{l}16.9 \% \\
31 / 183\end{array}$ & $\begin{array}{l}12.9 \% \\
13 / 101\end{array}$ & $\begin{array}{l}17.1 \% \\
20 / 117\end{array}$ & $\begin{array}{l}19.6 \% \\
20 / 102\end{array}$ & $<0.01 *$ \\
\hline $\begin{array}{l}\text { Labia majora } \\
\text { augmentation }\end{array}$ & $\begin{array}{l}72.1 \% \\
132 / 183\end{array}$ & $\begin{array}{l}41.6 \% \\
42 / 101\end{array}$ & $\begin{array}{l}75.2 \% \\
88 / 117\end{array}$ & $\begin{array}{l}51 \% \\
52 / 102\end{array}$ & $\begin{array}{l}27.3 \% \\
50 / 183\end{array}$ & $\begin{array}{l}50.5 \% \\
51 / 101\end{array}$ & $\begin{array}{l}18.8 \% \\
22 / 117\end{array}$ & $\begin{array}{l}49 \% \\
50 / 102\end{array}$ & $\begin{array}{l}0.5 \% \\
1 / 183\end{array}$ & $\begin{array}{l}7.9 \% \\
8 / 101\end{array}$ & $\begin{array}{l}6 \% \\
7 / 117\end{array}$ & $\begin{array}{l}0 \% \\
0 / 102\end{array}$ & $<0.01^{*}$ \\
\hline $\begin{array}{l}\text { Labia majora } \\
\text { reduction }\end{array}$ & $\begin{array}{l}71 \% \\
130 / 183\end{array}$ & $\begin{array}{l}43.6 \% \\
44 / 101\end{array}$ & $\begin{array}{l}76.1 \% \\
89 / 117\end{array}$ & $\begin{array}{l}40.2 \% \\
41 / 102\end{array}$ & $\begin{array}{l}27.9 \% \\
51 / 183\end{array}$ & $\begin{array}{l}51.5 \% \\
52 / 101\end{array}$ & $\begin{array}{l}19.7 \% \\
23 / 117\end{array}$ & $\begin{array}{l}57.8 \% \\
59 / 102\end{array}$ & $\begin{array}{l}1.1 \% \\
2 / 183\end{array}$ & $\begin{array}{l}5 \% \\
5 / 101\end{array}$ & $\begin{array}{l}4.3 \% \\
5 / 117\end{array}$ & $\begin{array}{l}2 \% \\
2 / 102\end{array}$ & $<0.01^{*}$ \\
\hline $\begin{array}{l}\text { Labia minora } \\
\text { augmentation }\end{array}$ & $\begin{array}{l}71.6 \% \\
131 / 183\end{array}$ & $\begin{array}{l}43.6 \% \\
44 / 101\end{array}$ & $\begin{array}{l}70.9 \% \\
83 / 117\end{array}$ & $\begin{array}{l}48 \% \\
49 / 102\end{array}$ & $\begin{array}{l}26.8 \% \\
49 / 183\end{array}$ & $\begin{array}{l}50.5 \% \\
51 / 101\end{array}$ & $\begin{array}{l}23.1 \% \\
27 / 117\end{array}$ & $\begin{array}{l}52 \% \\
53 / 102\end{array}$ & $\begin{array}{l}1.6 \% \\
3 / 183\end{array}$ & $\begin{array}{l}5.9 \% \\
6 / 101\end{array}$ & $\begin{array}{l}6 \% \\
7 / 117\end{array}$ & $\begin{array}{l}0 \% \\
0 / 102\end{array}$ & $<0.01^{*}$ \\
\hline $\begin{array}{l}\text { Labia minora } \\
\text { reduction }\end{array}$ & $\begin{array}{l}72.1 \% \\
132 / 183\end{array}$ & $\begin{array}{l}43.6 \% \\
44 / 101\end{array}$ & $\begin{array}{l}72.6 \% \\
85 / 117\end{array}$ & $\begin{array}{l}46.1 \% \\
47 / 102\end{array}$ & $\begin{array}{l}27.3 \% \\
50 / 183\end{array}$ & $\begin{array}{l}50.5 \% \\
51 / 101\end{array}$ & $\begin{array}{l}20.5 \% \\
24 / 117\end{array}$ & $\begin{array}{l}52 \% \\
53 / 102\end{array}$ & $\begin{array}{l}0.5 \% \\
1 / 183\end{array}$ & $\begin{array}{l}5.9 \% \\
6 / 101\end{array}$ & $\begin{array}{l}6.8 \% \\
8 / 117\end{array}$ & $\begin{array}{l}2 \% \\
2 / 102\end{array}$ & $<0.01 *$ \\
\hline Vaginoplasty & $\begin{array}{l}74.9 \% \\
137 / 183\end{array}$ & $\begin{array}{l}49.5 \% \\
50 / 101\end{array}$ & $\begin{array}{l}79.5 \% \\
93 / 117\end{array}$ & $\begin{array}{l}53.9 \% \\
55 / 102\end{array}$ & $\begin{array}{l}24 \% \\
44 / 183\end{array}$ & $\begin{array}{l}46.5 \% \\
47 / 101\end{array}$ & $\begin{array}{l}16.2 \% \\
19 / 117\end{array}$ & $\begin{array}{l}46.1 \% \\
47 / 102\end{array}$ & $\begin{array}{l}1.1 \% \\
2 / 183\end{array}$ & $\begin{array}{l}4 \% \\
4 / 101\end{array}$ & $\begin{array}{l}4.3 \% \\
5 / 117\end{array}$ & $\begin{array}{l}0 \% \\
0 / 102\end{array}$ & $<0.01 *$ \\
\hline $\begin{array}{l}\text { Laser vaginal } \\
\text { tightening }\end{array}$ & $\begin{array}{l}73.2 \% \\
134 / 183\end{array}$ & $\begin{array}{l}42.6 \% \\
43 / 101\end{array}$ & $\begin{array}{l}75.2 \% \\
88 / 117\end{array}$ & $\begin{array}{l}39.2 \% \\
40 / 102\end{array}$ & $\begin{array}{l}26.2 \% \\
48 / 183\end{array}$ & $\begin{array}{l}50.5 \% \\
51 / 101\end{array}$ & $\begin{array}{l}18.8 \% \\
22 / 117\end{array}$ & $\begin{array}{l}58.8 \% \\
60 / 102\end{array}$ & $\begin{array}{l}0.5 \% \\
1 / 183\end{array}$ & $\begin{array}{l}6.9 \% \\
7 / 101\end{array}$ & $\begin{array}{l}6 \% \\
7 / 117\end{array}$ & $\begin{array}{l}2 \% \\
2 / 102\end{array}$ & $<0.01 *$ \\
\hline $\begin{array}{l}\text { Laser for } \\
G^{\prime} M^{a}\end{array}$ & $\begin{array}{l}75.4 \% \\
138 / 183\end{array}$ & $\begin{array}{l}43.6 \% \\
44 / 101\end{array}$ & $\begin{array}{l}75.2 \% \\
88 / 117\end{array}$ & $\begin{array}{l}51 \% \\
52 / 102\end{array}$ & $\begin{array}{l}24 \% \\
44 / 183\end{array}$ & $\begin{array}{l}51.5 \% \\
52 / 101\end{array}$ & $\begin{array}{l}17.1 \% \\
20 / 117\end{array}$ & $\begin{array}{l}47.1 \% \\
48 / 102\end{array}$ & $\begin{array}{l}0.5 \% \\
1 / 183\end{array}$ & $\begin{array}{l}5 \% \\
5 / 101\end{array}$ & $\begin{array}{l}7.7 \% \\
9 / 117\end{array}$ & $\begin{array}{l}2 \% \\
2 / 102\end{array}$ & $<0.01 *$ \\
\hline $\begin{array}{l}\text { Vulvar/ } \\
\text { perianal } \\
\text { bleaching }\end{array}$ & $\begin{array}{l}69.4 \% \\
127 / 183)\end{array}$ & $\begin{array}{l}38.6 \% \\
39 / 101\end{array}$ & $\begin{array}{l}71.8 \% \\
84 / 117\end{array}$ & $\begin{array}{l}49 \% \\
50 / 102\end{array}$ & $\begin{array}{l}27.3 \% \\
50 / 183\end{array}$ & $\begin{array}{l}56.4 \% \\
57 / 101\end{array}$ & $\begin{array}{l}20.5 \% \\
24 / 117\end{array}$ & $\begin{array}{l}48 \% \\
49 / 102\end{array}$ & $\begin{array}{l}3.3 \% \\
6 / 183\end{array}$ & $\begin{array}{l}5 \% \\
5 / 101\end{array}$ & $\begin{array}{l}7.7 \% \\
9 / 117\end{array}$ & $\begin{array}{l}2.9 \% \\
3 / 102\end{array}$ & $<0.01^{*}$ \\
\hline Liposculpture & $\begin{array}{l}71.6 \% \\
131 / 183\end{array}$ & $\begin{array}{l}41.6 \% \\
42 / 101\end{array}$ & $\begin{array}{l}69.2 \% \\
81 / 117\end{array}$ & $\begin{array}{l}47.1 \% \\
48 / 102\end{array}$ & $\begin{array}{l}27.9 \% \\
51 / 183\end{array}$ & $\begin{array}{l}52.5 \% \\
53 / 101\end{array}$ & $\begin{array}{l}25.6 \% \\
30 / 117\end{array}$ & $\begin{array}{l}46.1 \% \\
47 / 102\end{array}$ & $\begin{array}{l}0.5 \% \\
1 / 183\end{array}$ & $\begin{array}{l}5.9 \% \\
6 / 101\end{array}$ & $\begin{array}{l}5.1 \% \\
6 / 117\end{array}$ & $\begin{array}{l}6.9 \% \\
7 / 102\end{array}$ & $<0.01^{*}$ \\
\hline
\end{tabular}

amplification. The majority of the participants agreed on the effect of FGCPs on improving the quality of life, improving self-esteem, and sexual functions of women. Near half of the respondents thought that the advertising and encouragement of FGCPs should be forbidden and were indecisive about whether FGCPs were genital mutilation.

In 2013, the Royal College of Obstetricians and Gynaecologists published an ethical opinion paper and pointed out that "the presentation of female genital cosmetic surgery (FGCS) as an unproblematic lifestyle choice is undesirable because it misleads women as to the need for and the efficacy of such surgical techniques" and stated that FGCS should not be undertaken unless it was medically indicated ${ }^{(7)}$. In July 2018, the United States Food and Drug Administration issued a warning against the use of energy-based devices outside of standardized research protocols for cosmetic vaginal procedures or vaginal "rejuvenation," citing their potential complications, including vaginal burns/scarring, dyspareunia, and chronic pain $^{(8)}$. In January 2020, the American College of Obstetricians and Gynecologists (ACOG) offered the term FGCS only for procedures "not medically indicated" and defined FGCS as "surgical alteration of the vulvovaginal anatomy intended for cosmesis in women who have no apparent structural or functional abnormality". The ACOG also recommended informing women about the lack of high-quality data supporting the effectiveness of genital cosmetic surgical procedures and their potential complications ${ }^{(9)}$.

For most FGCPs including hymenoplasty, G-spot augmentation, and clitoral hood reduction, the majority of the participants stated that there was rarely medical justification to perform the 


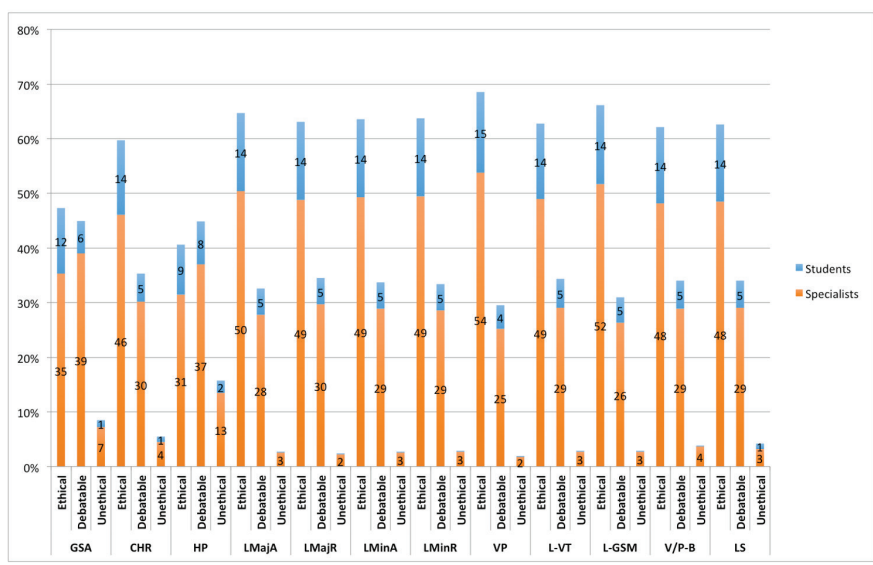

Figure 2. Perceptions of participants in terms of medical ethics

GSA: G-spot amplification, CHR: Clitoral hood reduction, HP: Hymenoplasty, LMajA: Labia majora augmentation, LMajR: Labia majora reduction, LMinA: Labia minora augmentation; LMinR: Labia minora reduction, VP: Vaginoplasty, L-VT: Laser vaginal tightening, L-GSM: Laser for genitourinary syndrome, V/P-B: Vulvar/perinal bleaching, LS: Liposculpturing

procedures despite recommendations of specialty committees. Although there is a lack of data to support the medical justification, especially for hymenoplasty, it is debatable if it can be performed because of religious reasons, after sexual abuse or preventing "honor" killings. According to the current survey, nearly half of the participants (49.3\%) found reasonable of a woman's need for hymenoplasty originated from social oppression. It can be lifesaving for a woman in Muslim societies, and it can be demanded to revive a sexual life by another woman living in another society ${ }^{(10)}$. On the other hand, authorities have concerns about violating women's rights and perpetuating human rights abuses ${ }^{(6)}$. The indication for G-spot procedures is also controversial because anatomic, radiologic, and biochemical studies regarding the G-spot have failed to provide evidence of its existence ${ }^{(11)}$. In the current survey, $52.7 \%$ of the participants considered that the G-spot existed but $52.8 \%$ found the procedure debatable/unethical. The procedure that participants found most ethically controversial was hymenoplasty, with $59.7 \%$ of the participants stating that hymenoplasty was debatable or unethical.

The participants considered that FGCPs could be performed, only upon patient request without medical indications. Although autonomy is the most important principle of medical ethics, patient requests could be ignored if the procedure is against "non-maleficence"(9). Patients who use autonomy should have sufficient knowledge about the procedure including scientific data about outcomes, complications, and comparisons of results with non-intervention ${ }^{(12)}$.

"The International Society for the Study of Vulvovaginal Disease" (ISSVD) stated that genital surgeons should determine whether the patient is competent to make medical decisions as a first step and recommended psychological counseling to all women who were considering FGCPs to give them a chance to express undisclosed thoughts and feelings $s^{(4,13,14)}$. Body dysmorphic disorder (BDD) is another entity that should be considered during preoperative evaluation ${ }^{(15)}$. The prevalence of BDD was determined as $53.6 \%$ in patients demanding esthetic surgery and $61.1 \%$ in patients demanding genital cosmetic surgeries. In our cohort, although nearly half (44.8\%) of the respondents supported psychological counseling before surgery, disagreement with this statement was highest among gynecologists.

The demand for FGCPs has been increasing in adults and teenagers ${ }^{(8)}$. Reaching adequate mental maturity is important for the patient to make rational decisions and also genital maturity should be provided to examine "normality" objectively. The Royal Australian College of General Practitioners, the ACOG, and the ISSVD recommend that FGCS should not be performed on girls aged younger than 18 years ${ }^{(4,16-18)}$. In the current survey, $81 \%$ of the participants also supported not performing FCGPs on girls aged under 18 years, irrespective of consent.

Just over half (54.5\%) of the participants agreed on the effect of FGCPs on improving the quality of life, $55.4 \%$ on improving selfesteem, and $54.1 \%$ on improving sexual functions of women. While $25.4 \%$ of the study group considered that FGCPs were only a temporary trend, $32.6 \%$ thought the opposite. Some authors suggested that labiaplasty and vaginal tightening could improve sexual function and quality of life, whereas others failed to demonstrate improvement ${ }^{(19-22)}$. In January 2020, the ACOG stated in their revised bulletin regarding FGCPs that surgical alteration of the labia that was not necessary to the health of patients aged younger than 18 years was a violation of federal criminal law in the United States ${ }^{(9,23)}$. The World Health Organization defined female genital mutilation as "all procedures involving partial or total removal of the external female genitalia or other injuries to the female genital organs for non-medical reasons" and this statement also raises concern about whether genital cosmetic surgeries constitute genital mutilation $^{(24)}$. Compatible with this discussion, nearly half of the participants (47\%) were indecisive and $19.1 \%$ considered FGCPs as genital mutilation. In surgical specialties, the rate of disagreeing with the idea that genital cosmetic surgeries are genital mutilation was higher, while the opposite was raised in non-surgical specialties.

In many business areas, especially thanks to the fact that social media is also in our lives and the number of active users is increasing rapidly, professionals are free to advertise their work. However, advertising is not clear-cut in medical practice because of moral, ethical and deontologic concerns. Almost half of the participants (47\%) did not find advertising suitable in terms of FGPCs. However, when the specialists' responses were examined independently, the group with the highest proportion of participants who thought that advertising could be used by cosmetic surgeons, was gynecologists. 
Table 3. Participants opinions about speculative comments regarding female genital cosmetic procedures

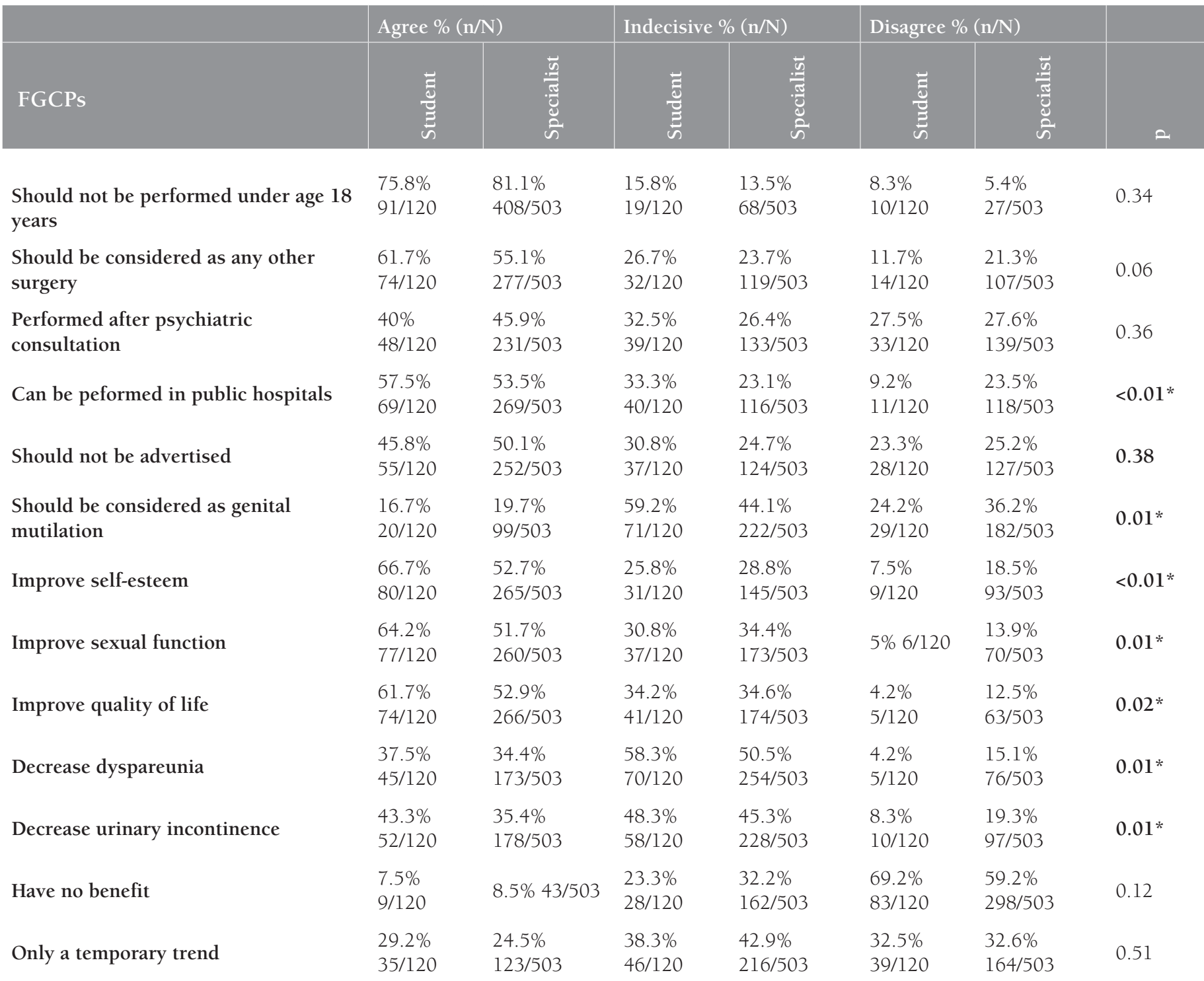

\section{Study Limitations}

\section{Ethics}

The weak point of this survey is that all of the physicians who answered the questionnaire were working in public hospitals. Although the opinions of physicians working in the private sector may affect the results, the perceptions/attitudes of physicians working in public hospitals may be more objective because they have no conflicts of interests.

\section{Conclusion}

The majority of participants declared that FGCPs could be performed only upon patient request and improved self-esteem, quality of life, and sexual functions. The most controversial procedures in terms of ethics were hymenoplasty and G-spot amplification. As the recommendations on the FGCPs are insufficient to define the boundaries of medical justification, genital mutilation, advertising, and ethical concerns, detailed guidelines for the protection of both patients and physicians are needed.
Ethics Committee Approval: The study was approved by the Institutional Review Board (E1/180/2019).

Informed Consent: The respondents were informed and consent for participation was obtained before administering the questionnaire.

Peer-review: Externally and internally peer-reviewed.

\section{Authorship Contributions}

Concept: G.F.Y., G.K., E.E.T., Design: G.F.Y., G.K., E.İ.S., Data Collection or Processing: G.F.Y., G.K., E.İ.S., İB.B., Analysis or Interpretation: H.L.K., Literature Search: H.L.K., Writing: G.F.Y., G.K., A.F.Y.

Conflict of Interest: The authors report no conflict of interest. Financial Disclosure: Authors have no financial interests about the research. 


\section{References}

1. Schick VR, Rima BN, Calabrese SK. Evulvalution: the portrayal of women's external genitalia and physique across time and the current Barbie doll ideas. J Sex Res 2011;48:74-81.

2. Ellibeș Kaya A, Doğan O, Yassa M, Başbuğ A, Özcan C, Çalışkan E. Do external female genital measurements affect genital perception and sexual function and orgasm? Turk J Obstet Gynecol 2020;17:175-81.

3. Nejadsarvari N, Ebrahimi A, Ebrahimi A, Hashem-Zade H. Medical ethics in plastic surgery: a mini review. World J Plast Surg 2016;5:207-12.

4. Vieira-Baptista P, Almeida G, Bogliatto F, Bohl TG, Burger M, Cohen-Sacher B, et al. International Society for the study of vulvovaginal disease recommendations regarding female cosmetic genital surgery. Low Genit Tract Dis 2018;22:415-34.

5. Vieira-Baptista P, Lima-Silva J, Fonseca-Moutinho J, Monteiro V, Águas F. Survey on aesthetic vulvovaginal procedures: what do portuguese doctors and medical students think? Rev Bras Ginecol Obstet 2017;39:415-23.

6. Leye E, Ogbe E, Heyerick M. 'Doing hymen reconstruction': an analysis of perceptions and experiences of Flemish gynaecologists. BMC Womens Health 2018;18:91.

7. Royal Collage of Obstetricians and Gynecologists. Ethical considerations in related to female cosmetic genital surgery. Ethical Opinion Paper; October 2013.

8. FDA Warns Against Use of Energy-Based Devices to Perform Vaginal 'Rejuvenation' or Vaginal Cosmetic Procedures: FDA Safety Communication; July 2018.

9. American College of Obstetricians and Gynecologists. ACOG Committee Opinion No. 795. Elective female genital cosmetic surgery. Obstet Gynecol 2020;135:36-42.

10. Ahmadi A. Ethical issues in hymenoplasty: views from Tehran's physicians. J Med Ethics 2014:40:429-30.

11. Singh A, Swift S, Khullar V, Digesu A. Laser vaginal rejuvenation: not ready for prime time. Int Urogynecol J 2015;26:163-4.

12. Goldstein A, Jutrzonka S. Ethical considerations of female genital plastic/cosmetic surgery. In: Goodman MP, editor. Female genital plastic and cosmetic surgery. 1st ed. Hoboken, New Jersey: John Wiley \& Sons Ltd; 2016:39-44.

13. Cantor JD. When an adult female seeks ritual genital alteration: ethics, law and the parameters of participation. Plast Reconstr Surg 2006;117:1158-64.
14. Barbara G, Facchin F, Meschia M, Vercellini P. "The first cut is the deepest": a psychological, sexological and gynecological perspective on female genital cosmetic surgery. Acta Obstet Gynecol Scand 2015;94:915-20.

15. Committee on Gynecologic Practice, American College of Obstetricians and Gynecologists. ACOG Committee Opinion No. 378: Vaginal "rejuvenation" and cosmetic vaginal procedures. Obstet Gynecol 2007;110:737-8.

16. The Royal Australian College of General Practitioners; Female genital cosmetic surgery - a resource for general practitioners and other health professionals. Melbourne: The Royal Australian College of General Practitioners; 2015.

17. Runacres SA, Wood PL. Cosmetic labiaplasty in an adolescent population. J Pediatr Adolesc Gynecol 2016; 29:218-22.

18. Westermann LB, Oakley SH, Mazloomdoost D, Crisp CC, Kleeman SD, Benbouajili JM, et al. Attitudes regarding labial hypertrophy and labiaplasty: a survey of members of the Society of Gynecologic Surgeons and the North American Society for Pediatric and Adolescent Gynecology. Female Pelvic Med Reconstr Surg 2016;22:175-9.

19. González-Isaza P, Lotti T, França K, Sanchez-Borrego R, Tortola JE, Lotti J, et al. Carbon dioxide with a new pulse profile and shape: a perfect tool to perform labiaplasty for functional and cosmetic purpose. Open Access Maced J Med Sci 2018;6:25-7.

20. Sharp G, Tiggemann M, Mattiske J. A retrospective study of the psychological outcomes of labiaplasty. Aesthet Surg J 2017;37:32431.

21. Veale D, Naismith I, Eshkevari E, Ellison N, Costa A, Robinson D, et al. Psychosexual outcome after labiaplasty: a prospective casecomparison study. Int Urogynecol J 2014;25:831-9.

22. İnan C, Ağır Çağrı M, Sağır Gökdağlı F, Özer A, Özbek Ö, Dayanır $\mathrm{H}$, et al. Assessment of the effects of perineoplasty on female sexual function. Balkan Med J 2015;32:260-5.

23. Ostrzenski A. Vaginal rugation rejuvenation (restoration): a new surgical technique for an acquired sensation of wide/smooth vagina. Gynecol Obstet Invest 2012;73:48-52.

24. Female genital mutilation. World Health Organisation (WHO) - Fact sheets. Last Accessed Date: 10.10.2020. Available from: https://www.who.int/health-topics/female-genital-mutilation. 